\title{
DiffErenCes in AntihypertenSive Drug Blood Levels in Patients with HypertensiON (DECISION): Protocol for a Prospective Observational Study Comparing Pharmacokinetics and Pharmacodynamics Between Young and Elderly Patients
}

\author{
Dimokrat Hassan ${ }^{1}$ (D) Laura E. J. Peeters ${ }^{1,2}$ - Birgit C. P. Koch ${ }^{1}$. Jorie Versmissen ${ }^{1,2}$
}

Received: 13 October 2021 / Accepted: 21 January 2022 / Published online: 17 February 2022

(c) The Author(s) 2022

\begin{abstract}
Adequate controlled blood pressure decreases the risk of cardiovascular events. However, the elderly are more vulnerable and thereby more prone to side effects of antihypertensive drugs. A lack of pharmacokinetic and pharmacodynamic (PK/ PD) studies in older patients makes specific and tailored advices towards antihypertensive drug therapy difficult. The aim of our study, DiffErenCes In antihypertenSive drug levels In patients with hypertensiON (DECISION), is to fill in this PK/ PD knowledge gap and move towards precision dosing. DECISION is a prospective observational PK/PD study set up to determine the difference in exposure to the antihypertensive drugs, losartan and perindopril, measured by drug levels in blood. The area under the curve (AUC; PK) and furthermore the association between the AUC and the effect on blood pressure (PD) will be compared between elderly and younger patients.
\end{abstract}

Keywords Hypertension · Antihypertensive agents $\cdot$ Humans $\cdot$ Aged $\cdot$ Pharmacokinetics

\section{Introduction}

The worldwide prevalence of hypertension is increasing, not the least because of an ageing population [1-3]. Older patients have a relative high prevalence of hypertension, which is an important risk factor for cardiovascular diseases

Dimokrat Hassan and Laura E. J. Peeters have an equal contribution to the DECISION study and the DECISION protocol.

Dimokrat Hassan

d.hassan@erasmusmc.nl

Laura E. J. Peeters

1.e.j.peeters@erasmusmc.nl

Birgit C. P. Koch

b.koch@erasmusmc.nl

Jorie Versmissen

j.versmissen@erasmusmc.nl

1 Department Hospital Pharmacy, Erasmus University Medical Center, Erasmus MC, University Medical Center Rotterdam, Postbus 2040, 3000 CA Rotterdam, The Netherlands

2 Department of Internal Medicine, Erasmus MC, University Medical Center Rotterdam, Rotterdam, The Netherlands
(CVD) $[3,4]$. In addition, they often have other comorbidities, like diabetes, further contributing to the risk for CVD [3].

Recently published prospective studies like the HYVET and the SPRINT trials emphasized that blood pressure control in older patients is beneficial, as long as treatment is well tolerated $[5,6]$.

Over the years, attention for negative side effects of (antihypertensive) drugs, especially in the frail elderly patients, increased. These side effects have led to an increased number of hospital admissions related to medication (HARM) and a significant decrease in quality of life among older patients [7-9].

It is assumed that, because of altered pharmacokinetic parameters, older patients have higher plasma concentrations of antihypertensive drugs compared to younger patients, when taking the same dose $[1,10]$. Furthermore, it has been shown that because of a decreased renin-angiotensinaldosterone System (RAAS) activity, the effect of antihypertensive drugs affecting RAAS is also decreased [1]. This could lead to the impulse of physicians to increase the dose of antihypertensive drugs because of a lack of effect, thereby 
increasing the risk of side effects which in turn could lead to non-adherence and undertreatment $[1,11,12]$.

The effects of ageing on the pharmacodynamics (PD) of antihypertensive drugs in terms of decrease in blood pressure are seldom studied and studies combining both PK/PD are even more scarce. To address this knowledge gap we set up the DECISION-study that compared the PK/PD of older patients ( $>70$ years) and younger patients ( $<50$ years) using the antihypertensive drugs losartan and perindopril. The results of this study will confirm if there is indeed a difference in plasma drug concentrations between the elderly and younger patients and if these differences are related to a relevant difference in blood pressure response.

\section{Aim}

The aim of the DECISION study is to address the PK/PD knowledge gap of antihypertensive drug in older patients. Better knowledge about pharmacokinetics (PK) and the relation between PK and PD could help determine more specific and tailored therapy, that will better fit older patients.

\section{Methods}

DECISION is a prospective observational study comparing the PK and PD between elderly and younger patients, which is approved by the Medical Ethical Committee of Erasmus MC. Patients will be screened and included from Erasmus Medical Centre (MC) Rotterdam and various general practices in the Netherlands.

Perindopril and losartan are the drugs of choice for this study, because they are frequently used antihypertensive drugs in the Netherlands. Furthermore, a patient friendly sampling method in the form of dried blood spot (DBS), which can be performed by means of a finger prick by the patients at home. Drug concentrations from the DBS are measured with a validated ultra-performance liquid chromatography-tandem mass spectrometry (UPLC-MS/MS method) and includes both drugs of interest and their active metabolites [13].

Patients aged 18 to 50 years and patients aged 70 years or older, using losartan or perindopril will be screened in the information systems of general practices and hospitals, using a search query. Also, physicians will be asked to include patients for whom they will prescribe either losartan or perindopril. There are no dose restrictions for perindopril or losartan.

Patients using any other antihypertensive drug that influences RAAS, beside losartan or perindopril, will be excluded. Other exclusion criteria are; patients with endstage renal disease (eGFR $<15 \mathrm{ml} / \mathrm{min}$ ) and those patients who cannot safely withdraw from their antihypertensive drugs for two weeks.

After giving informed consent, measurements will be carried out over two testing days, with an interval of at least 2 weeks between the 2 days to assure a steady state or total absence of perindopril and losartan in blood.

On one of the testing days a baseline blood pressure measurement will be performed, without the use of perindopril or losartan. For this baseline measurement patients are asked to not use the antihypertensive drug for a consecutive period of at least 2 weeks up to and including the testing day.

On the other testing day, measurements will be performed when the patient is using the antihypertensive drug for at least 2 weeks. Blood pressure will be measured and simultaneously blood will be sampled by means of the DBS method at the following intervals after drug intake; before intake of the drug $(\mathrm{t}=0), 1 \mathrm{~h}$ after intake of the drug $(\mathrm{t}=1 \mathrm{~h})$ and 4,6 and $24 \mathrm{~h}$ after intake of the $\operatorname{drug}(\mathrm{t}=4 \mathrm{~h}, \mathrm{t}=6 \mathrm{~h}, \mathrm{t}=24 \mathrm{~h})$. A schematic overview of this study design can be found in Fig. 1.

\section{Expected Results}

The blood pressure will be measured using 24-h ambulatory blood pressure measurements (24-h ABPM) and blood samples will be collected at home using DBS [13]. The DBS will be used to measure drug concentrations of losartan and perindopril and their active metabolites at five different time points. Thereafter, the Area Under the Curve (AUC) for the parent drug and metabolites will be calculated.

The follow-up period is set to 4 weeks, wherein patients can write down adverse events in a diary. Only adverse events related to antihypertensive drug therapy or the temporary ceasing of antihypertensive drug therapy will be included in the DECISION-study.

Furthermore, the following parameters will be collected for each patient at baseline: age, sex, BMI $\left(\mathrm{kg} / \mathrm{m}^{2}\right)$, kidney function, comorbidities including CVD, heart failure, Chronic Obstructive Pulmonary Disease (COPD), diabetes and other medication by Anatomical Therapeutic Chemical classification (ATC-code) and dose.

During the research and in the follow-up period, patients will be asked to report all adverse events (AE) and serious adverse events (SAE).

\subsection{Statistical Analysis}

For the primary objective, the AUCs of the antihypertensive drugs per patient are calculated using non compartmental analysis and thereafter corrected for the dose. The AUC averages of older and younger patients are log transformed aiming for a normal distribution. These averages per group 


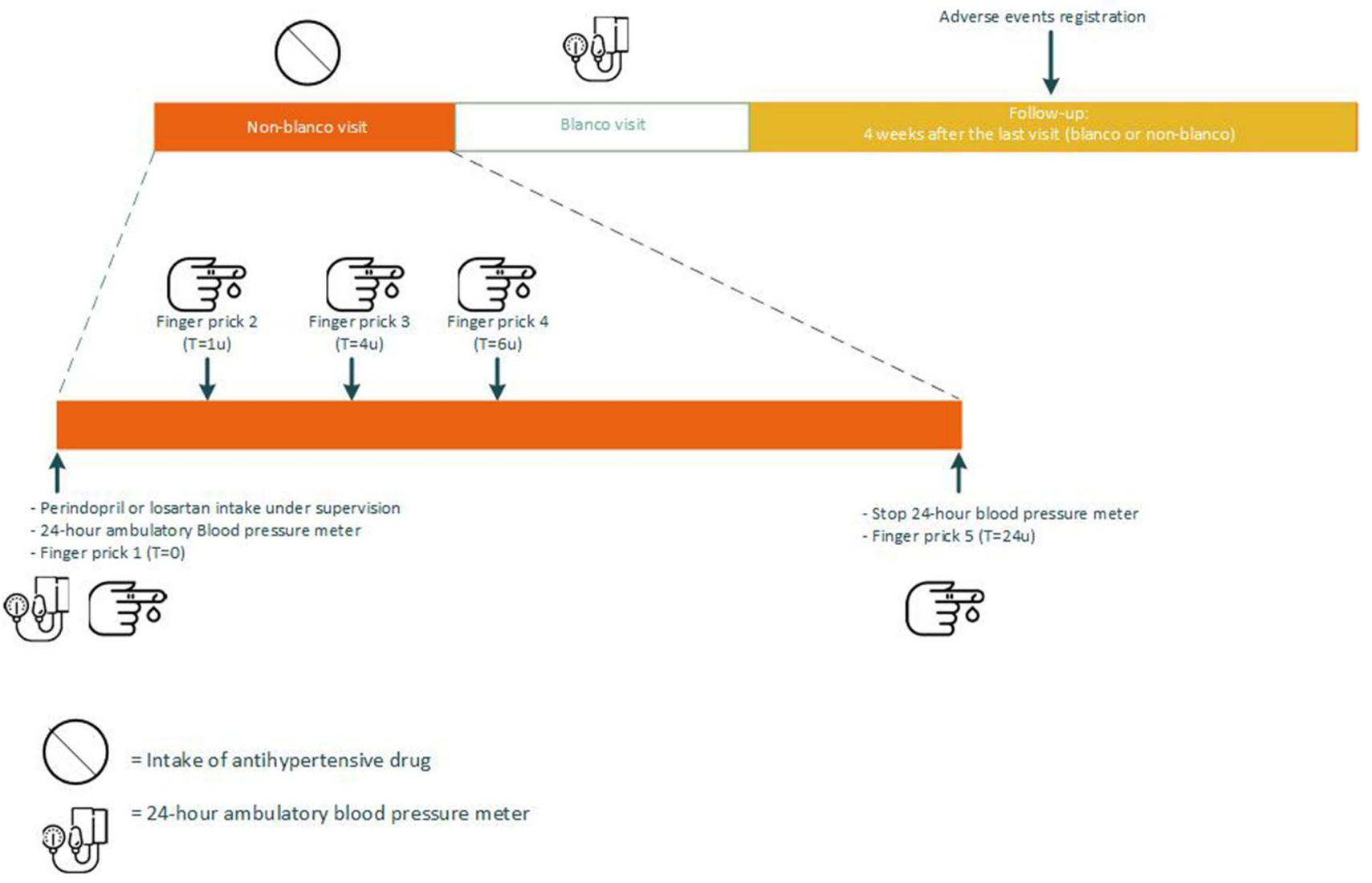

Fig. 1 schematic overview of the DECISION study design

are then compared and tested by means of an unpaired sample t-test. If the data is not normally distributed after log transformation, a non-parametric test will be used to calculate the difference in AUC.

The difference in the average 24-h and daytime blood pressure before and after the use of perindopril or losartan between the elderly and younger patients is compared and tested by means of an unpaired sample t-test, when the data is normally distributed. If the data is not normally distributed, a non-parametric test will be used.

Linear regression will be applied to test the relation of the antihypertensive drug AUC and the average blood pressure.

Multiple linear regression analysis on the AUC will be performed to determine the role of the following covariates; age, sex, BMI and the use of multiple antihypertensive drugs.

\subsection{Sample Size Calculation}

The analyses for the AUC differences are based on logtransformed AUCs, reasonably assuming that these are normally distributed [14]. The mean and standard deviation (SD) of $\log (\mathrm{AUC})$ in the target populations are unknown. Therefore, the sample size calculation is based on Cohen's $\mathrm{d}$ effect size, i.e. the mean difference in $\log$ (AUC) between the patients $<50$ and those $>70$ years, divided by the SD of $\log$ (AUC), while assuming similar SDs in both populations that are equal to the expected difference. Hence, the expected effect size is equal to 1 regardless of the precise mean difference and SD. This effect size was considered as the minimal clinically relevant difference (MCRD). A total of 16 patients $<50$ and 16 patients $>70$ years for either perindopril or losartan are then required to reveal this MCRD with a power $(1-\beta)$ of $80 \%$, while performing a two-sided test with $\alpha=0.05$. In order to compensate for dropouts, we aim to enrol 20 patients in the two age categories $(<50$ and $>70$ years) per drug (losartan and perindopril), resulting in a combined sample size of 80 patients.

\section{Conclusion}

There is still a lot unknown about the PK/PD of antihypertensive drugs in older patients. A better knowledge of the relation between $\mathrm{PK}$ and $\mathrm{PD}$ of antihypertensive drugs can help move towards precision dosing, resulting in increased therapy effectiveness and decreased adverse drug events. Side effects due to an incorrect antihypertensive drug 
treatment form a barrier in an otherwise beneficial therapy for CVD. In daily practice, the therapy is adjusted to clinical outcomes. However, this is a reactive approach and it means the negative side effects have already occurred.

With the DECISION-study, we start with two commonly prescribed antihypertensive drugs, perindopril and losartan, as proof of principle. We are well aware that there is need for more PK/PD related knowledge of (all) other antihypertensive drugs. Perindopril and losartan are, because of the prevalent use and the availability of a well-validated and patient friendly DBS sampling method, excellent first candidates to study differences between older and younger patients.

While we anticipate valuable results and information from the DECISION-study, we also acknowledge some minor limitations of our set-up.

The variability in the DBS-method is a little bit higher than the commonly used blood sample measurement [13, 15]. Also, patients need a thorough instruction to correctly sample their blood on paper for the DBS sampling at home. Various previous studies investigated the feasibility of DBS and concluded that the benefits of DBS home measurements, like less visits to the hospital and lower costs, outweigh the minor limitations [16]. We therefore expect that because of reduced visits and lower patient burden, more patient are willing to participate.

Second, measuring blood pressure can also introduce variability on the PD-level, depending on the method of choice. To diminish this variability and increase uniform measurements, 24-h ABPM will be used, which is considered the golden standard to measure blood pressure. To gain more insight in potential external factors patients are asked to write down all factors that are of possible influence on their blood pressure.

Another point of discussion lies in our study population. We expect to include most of our young patients at Erasmus MC, which is a tertiary hospital. These patients are more likely to have received a kidney-transplantation or have secondary forms of hypertension. It is not clear if they will react the same way to antihypertensive drugs (PD) as elderly patients.

In conclusion, the DECISION study may have some minor limitations, but overall, the study set up is solid, feasible and has a low burden on patients.

\subsection{Clinical Expectations}

We expect to gain valuable information with the DECISION-study to fill in a PK/PD knowledge gap in older patients using perindopril or losartan. This information could help in clinical practice towards precision dosing and a more effective therapy in older patients. Although, existing guideline, like that of the European Society of Cardiologists [2], advice carefulness in dosing antihypertensive drugs in older patients, these advices are not backed by PK/PD data or are not specific enough for clinical care.

To our best knowledge, this is the first study to combine $\mathrm{PK} / \mathrm{PD}$ in a real-life population with the use of DBS to optimize antihypertensive drug therapy in the elderly patient.

Acknowledgements Our gratitude to H. Boersma, for his important contribution in the statistical methods section.

\section{Declarations}

Funding The DECISION study is supported by the Erasmus MC grant mRace with project number 2020-20207.

Conflict of interest All authors declare no conflicts of interest.

Open Access This article is licensed under a Creative Commons Attribution-NonCommercial 4.0 International License, which permits any non-commercial use, sharing, adaptation, distribution and reproduction in any medium or format, as long as you give appropriate credit to the original author(s) and the source, provide a link to the Creative Commons licence, and indicate if changes were made. The images or other third party material in this article are included in the article's Creative Commons licence, unless indicated otherwise in a credit line to the material. If material is not included in the article's Creative Commons licence and your intended use is not permitted by statutory regulation or exceeds the permitted use, you will need to obtain permission directly from the copyright holder. To view a copy of this licence, visit http://creativecommons.org/licenses/by-nc/4.0/.

\section{References}

1. Peeters LEJ, Kester MP, Feyz L, Van Den Bemt PMLA, Koch BCP, Van Gelder T, Versmissen J. Pharmacokinetic and pharmacodynamic considerations in the treatment of the elderly patient with hypertension. Expert Opin Drug Metab Toxicol. 2019;15(4):287-97. https://doi.org/10.1080/17425255.2019. 1588249.

2. Williams B, Mancia G, Spiering W, Agabiti Rosei E, Azizi M, Burnier M, et al. 2018 ESC/ESH Guidelines for the management of arterial hypertension: The Task Force for the management of arterial hypertension of the European Society of Cardiology (ESC) and the European Society of Hypertension (ESH). Eur Heart J. 2018;39(33):3021-104. https://doi.org/10.1093/eurhe artj/ehy339.

3. Lee J-H, Kim K-I, Cho M-C. Current status and therapeutic considerations of hypertension in the elderly. Korean J Intern Med. 2019;34(4):687-95. https://doi.org/10.3904/kjim.2019.196.

4. Nederlands Huisartsen Genootschap (NHG), 2019. Cardiovascular Risk Management (M84) [In Dutch]. NHG-guidelines. [online] Nederlands Huisartsen Genootschap (NHG). richtlijnen.nhg.org/ files/pdf/93_Cardiovasculair\%20risicomanagement_juni-2019. pdf. Accessed 19 May 2021.

5. Williamson JD, Supiano MA, Applegate WB, Berlowitz DR, Campbell RC, Chertow GM, Fine LJ, Haley WE, Hawfield AT, Ix JH, Kitzman DW, Kostis JB, Krousel-Wood MA, Launer LJ, Oparil S, Rodriguez CJ, Roumie CL, Shorr RI, Sink KM, Wadley $\mathrm{VG}$, et al. Intensive vs standard blood pressure control and cardiovascular disease outcomes in adults aged $\geq 75$ years: a randomized 
clinical trial. JAMA. 2016;315(24):2673-82. https://doi.org/10. 1001/jama.2016.7050.

6. Bulpitt CJ, Beckett NS, Cooke J, Dumitrascu DL, Gil-Extremera B, Nachev C, Nunes M, Peters R, Staessen JA. Lute on behalf of the Hypertension in the Very Elderly Trial (HYVET) Working Group Results of the pilot study for the Hypertension in the Very Elderly Trial. J Hypertension. 2003;21(12):2409-17.

7. Leendertse AJ, Egberts AC, Stoker LJ, van den Bemt PM, HARM Study Group. Frequency of and risk factors for preventable medication-related hospital admissions in the Netherlands. Arch Intern Med. 2008;168(17):1890-6. https://doi.org/10.1001/archintern med.2008.3.

8. Ministerie van Volksgezondheid, Welzijn en Sport. HARMWRESTLING: a proposal from the expert group medication safety concerning concrete interventions which will help make outpatient care safer in a short period of time [In Dutch]. Ministerie van Volksgezondheid, Welzijn en Sport; 2008.

9. Sturkenboom MCJM, et al. Follow-up Study on Medication Safety [in Dutch]. IPCI Erasmus MC Rotterdam, 2017.

10. Turnheim K. When drug therapy gets old: pharmacokinetics and pharmacodynamics in the elderly. Exp Gerontol. 2003;38(8):84353. https://doi.org/10.1016/s0531-5565(03)00133-5.

11. Abernethy DR, Gutkowska J, Winterbottom LM. Effects of amlodipine, a long-acting dihydropyridine calcium antagonist in aging hypertension: pharmacodynamics in relation to disposition. Clin Pharmacol Ther. 1990;48(1):76-86.

12. Sica DA. Dosage considerations with perindopril for systemic hypertension. Am J Cardiol. 2001;88(7A):13i-i18.

13. Peeters L, Feyz L, Hameli E, Zwart T, Bahmany S, Daemen J, et al. Clinical validation of a dried blood spot assay for 8 antihypertensive drugs and 4 active metabolites. Ther Drug Monit. 2019;42:460-7.

14. Keene ON. The log transformation is special. Stat Med. 1995;14(8):811-9. https://doi.org/10.1002/sim.4780140810.

15. van der Nagel BCH, Versmissen J, Bahmany S, van Gelder T, Koch BCP. High-throughput quantification of 8 antihypertensive drugs and active metabolites in human plasma using UPLCMS/MS. J Chromatogr B Analyt Technol Biomed Life Sci. 2017;15(1060):367-73. https://doi.org/10.1016/j.jchromb.2017. 06.029 .

16. Veenhof H, Boven J, Voort A, Berger S, Bakker S, Touw D. Effects, costs and implementation of monitoring kidney transplant patients' tacrolimus levels with dried blood spot sampling: a randomized controlled hybrid implementation trial. Br J Clin Pharmacol. 2020;86(7):1357-66. https://doi.org/10.1111/bcp. 14249 . 